\title{
Software Design of Ultrasonic Ranging System in Agricultural Robot
}

\author{
Zhang Fu ${ }^{1}$, Fu Sanling ${ }^{2}$, Liu Yong ${ }^{3}$, Zhang Yakun ${ }^{4}$ \\ ${ }^{1,4}$ (College of Vehicle \& Motive Power Engineering, Henan University of Science and Technology, Luoyang, $P$ \\ $R$ China) \\ ${ }_{2}^{2}$ (College of Physics and Engineering, Henan University of Science and Technology, Luoyang, P R China) \\ ${ }^{3}$ (Great Wall Motor Company Limited (GWM), Baoding, P R China)
}

\begin{abstract}
The software system of ultrasonic ranging system in agricultural robot was designed. This ultrasonic ranging system has two most components including the transmitter and the receiver. The ultrasonic ranging method was concluded, and ultrasonic ranging system generally calculates distance using the time-of-flight method, and the sound velocity must adjust by temperature compensation method when the precision of the ranging is a little high. The signal of $40 \mathrm{kHz}$ was generated by the single chip microprocessor, and amplified and transmitted by the amplifying circuit and the ultrasonic transmitter respectively. The ultrasonic signal was amplified by the amplifying circuit after the ultrasonic receiver received the signal, and detected by the phaselock loop circuit, and the time $t$ was measured by the interrupt program of the single chip microprocessor, finally, the distance was calculated by software discriminant. Ultrasonic ranging system software module structure mainly included main program module, the ultrasonic transmitting module, the interrupt service module of the comparator, the compensation module at room temperature and the display module. The result showed that the software system of ultrasonic ranging system can satisfy the function requirement of the agricultural robot.
\end{abstract}

Keywords - Agricultural robot, Ultrasonic ranging system, Software design, TOF method, Microcomputer

\section{INTRODUCTION}

Ultrasonic sensors have been used for many purposes in agriculture for more than 40 years. The distance measuring system must be equipped in order to make mobile robots walk automatically on the station of the obstacle, which can achieve timely the distance and the direction of the obstacle. Recognizing environments is the functions of mobile robots, which are required to find unpredictable obstacles and paths through which the robots can pass. The range sensors can measure a distance to objects, and ultrasonic sensor is more commonly used with mobile robots because it is small, inexpensive and easy to calculate distances. Ultrasonic ranging system is one of the most popular kinds of application on the ranging program between the combination of the sensor technology and the auto-control technology that is widely used in the security, the parking-assistance system, the construction site and other industrial fields. Ultrasonic wave has the advantages including the strong directional characteristics, slow energy consumption characteristics, far distance transmission characteristics, so often used for the measurement of the distance [1,2].

The real-time distance between the robot and the object must be measured on the walking process of the agricultural robot, and the exercise equipment and related equipment must make certain action when the distance achieve a certain range, thus complete automatically the specific operation. The distance between the robot and the end flag was measured when the agricultural robot walk nearly the end of the track, then the brake system or the reversing operation must play a role $[3,4]$. The non-contacting ranging must detect obstacles in order to avoid the obstacles or pass the obstacles when the agricultural robot walks on the rough road or the road with an obstacle, ultrasonic ranging system can meet effectively the detection distance of the agricultural robot, so realize the contact distance measurement and make corresponding action. In the paper, software design of ultrasonic ranging system in agricultural robot was researched based on hardware design.

\section{ULTRASONIC RANGING PRINCIPLE}

\subsection{Ultrasonic ranging method}

Ultrasonic ranging system generally calculates distance using the time-of-flight (TOF) method. The distance $s$ to a reflected object is calculated by

$s=v^{*} t / 2$,

Where $v$ is the speed of sound, and $t$ is the round-trip time-of-flight. The principle of the time-of-flight method was showed as Fig. 1. 


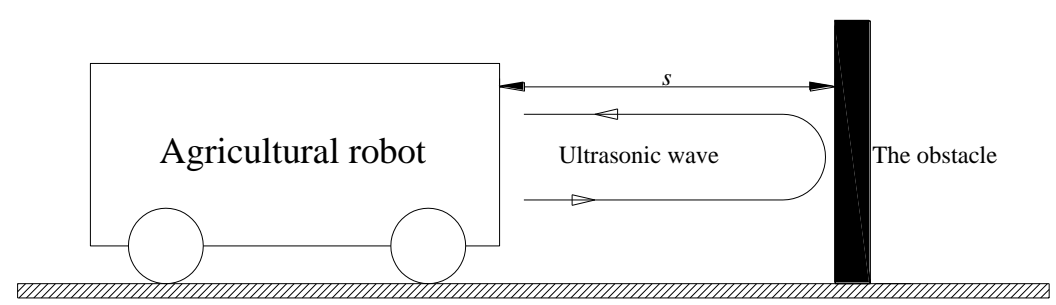

Fig. 1 the principle of the time-of-flight method

The TOF method will produce a range value when the echo amplitude first exceeds the threshold level after transmitting. The information obtained by the ultrasonic sensor is influenced by the characteristics of the sensing system and its environment. The sound velocity $v$ is related to the temperature because ultrasonic wave is also a kind of sound $[5,6]$. The relationship table between ultrasonic wave velocity and temperature was showed as Tab. 1. The sound velocity is invariable when the temperature is not significantly changed, but the sound velocity must adjust by temperature compensation method when the precision of the ranging is a little high.

Tab. 1 the relationship table between ultrasonic wave velocity and temperature

\begin{tabular}{ccccccccc}
\hline Temperature $\left({ }^{\circ} \mathrm{C}\right)$ & -30 & -20 & -10 & 0 & 10 & 20 & 30 & 100 \\
\hline Sound velocity $(\mathrm{m} / \mathrm{s})$ & 313 & 319 & 325 & 331 & 338 & 344 & 349 & 386 \\
\hline
\end{tabular}

That ultrasonic wave in the air velocity and temperature (unit: degrees Celsius) has the following approximate relations:

$V=V_{0}+0.607 T$

Where, $V_{0}$ is the acoustic velocity at $0^{\circ} \mathrm{C}$ for $331.45 \mathrm{~m} / \mathrm{s}$, and $\mathrm{T}$ is the actual temperature $\left({ }^{\circ} \mathrm{C}\right)$. Ultrasonic velocity will change about $0.6 \mathrm{~m} / \mathrm{s}$ with the temperature changing 1 degrees Celsius at room temperature, so the current temperature was measured through the temperature measurement circuit, which can be calculated ultrasonic transmission speed in the current temperature. The monobus digital temperature sensor DS18B20 was used for main components of temperature measurement circuit, which has more advantages with high precision and intelligence, small size and simple circuit. The relationship table between ultrasonic wave velocity and temperature after compensation was showed as Tab.2 [7]. As can be showed from Tab. 2, the sound velocity value is similar to the Tab. 1 under $0^{\circ} \mathrm{C}$, and the maximum error is no more than $5 \%$ above $0^{\circ} \mathrm{C}$, and the precision of the temperature directly affects of the speed of sound.

Tab. 2 the relationship table between ultrasonic wave velocity and temperature after compensation

\begin{tabular}{ccccccccc}
\hline Temperature $\left({ }^{\circ} \mathrm{C}\right)$ & -30 & -20 & -10 & 0 & 10 & 20 & 30 & 100 \\
\hline Sound velocity $(\mathrm{m} / \mathrm{s})$ & 313 & 319 & 325 & 331 & 337 & 343 & 349 & 381 \\
\hline
\end{tabular}

\section{2 the principle diagram of ultrasonic ranging system}

The signal of 40kHZ was generated by the single chip microprocessor, and amplified and transmitted by the amplifying circuit and the ultrasonic transmittor respectively. The ultrasonic signal was amplified by the amplifying circuit after the ultrasonic receiver received the signal, and dectected by the phase-lock loop circuit, and the time $t$ was measured by the interrupt program of the single chip microprocessor, finally, the distance was calculated by software discriminant. The principle diagram of ultrasonic ranging system was showed as Fig. 2.

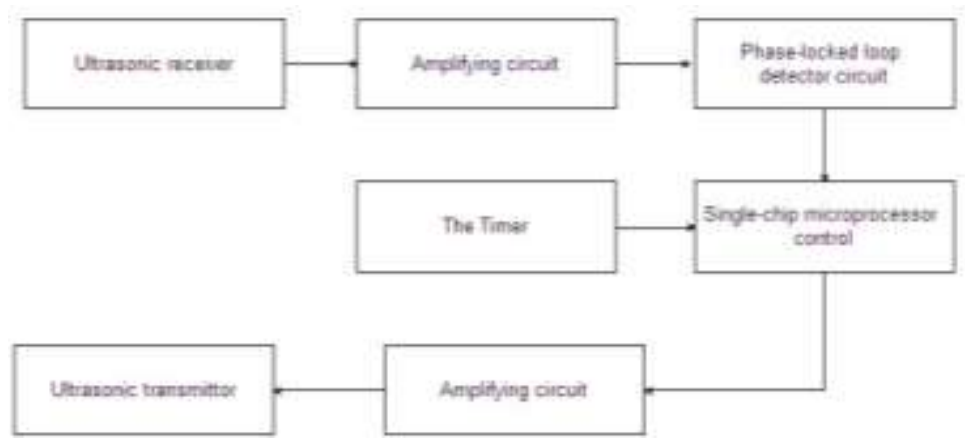

Fig. 2 the principle diagram of ultrasonic ranging system 


\section{The Structure Of The Hardware System}

This ultrasonic ranging system has two most components including the transmitter and the receiver. The main function of the work machine is accompanied by agricultural robot, and the distance between the robot and the object was measured, and the operator will receive the measuring distance through the transmitter. The receiver can collect the data of the transmission transmitter and show to the operator through the LED display. Each module in the ultrasonic ranging system structure was showed as Fig.3 [2].

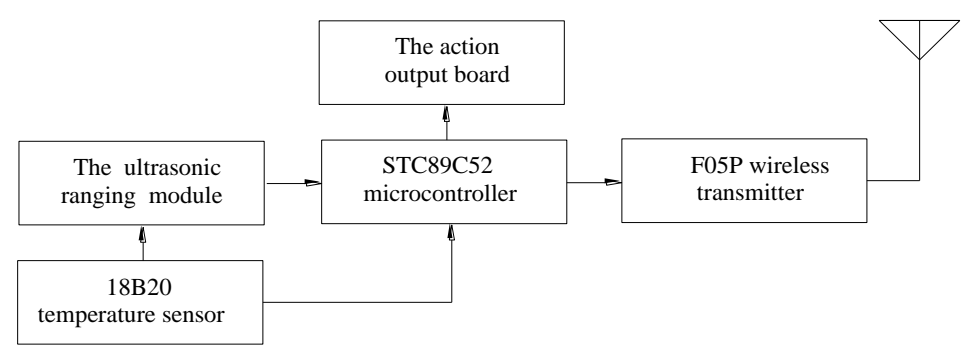

(1) the ultrasonic wave transmitter

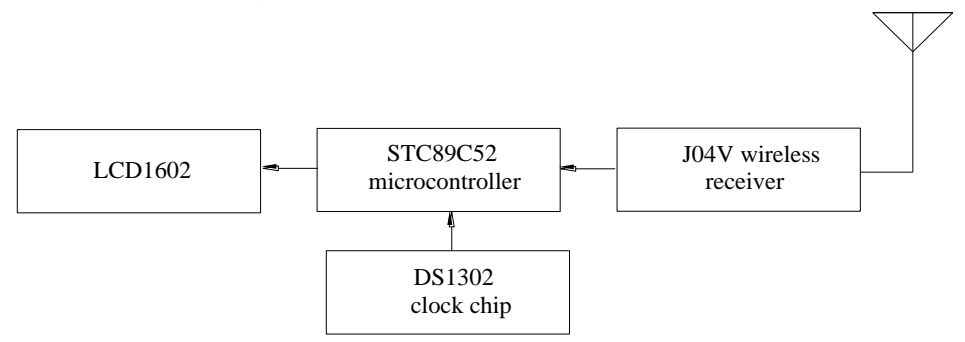

(2) the ultrasonic wave receiver

Fig. 3 The block diagram of the ultrasonic ranging system

STC89C52 microcontroller can control each module of the transmitter to complete their functions in sequence, and each module of the data processing will control the action output board to work, and the real-time data will be transmitted to the wireless launcher. 18B20 temperature sensor will measure the environmental temperature in real-time condition and the ultrasonic velocity will revise based on the relation with the temperature. The ultrasonic module will emit or receive ultrasonic wave to mark the timer by single-chip microcomputer control. The action output board will control relay action by the scheduled program when the distance is $50 \mathrm{~mm}, 100$ $\mathrm{mm}$ and $200 \mathrm{~mm}$ and $400 \mathrm{~mm}$. The F05P wireless transmitting module will transmit the data from chip serial port in the form of wireless signals.

The J04V wireless signal receiving module will receive the wireless signals, and send a signal to the microcontroller serial port according to the instructions of the single-chip microcomputer. The STC89C52 microcontroller will receive data for the display format form of LCD1602, and control each module of the transmitter in sequence on time to complete their functions, and each module is comprehensive treatment to LCD1602 to display. DS1302 clock chip will accurately provide real-time time included hour, minute, second, week, day, month and year. LCD1602 LCD monitor will display the distance, the temperature, the time and other data.

\section{THE SOFTWARE DESIGN OF THE SYSTEM}

Ultrasonic ranging system software module structure mainly included main program module, the ultrasonic transmitting module, the interrupt service module of the comparator, the compensation module at room temperature and the display module. Ultrasonic transmitting module will send $200 \mu$ s square wave pulse with $40 \mathrm{KHz}$, and control ultrasonic pulse by ultrasonic transmitter. The interrupt service module of the comparator controls the counter, which can realize echo time detection. The temperature analog voltage value will convert to digital value through the $\mathrm{ADC}$ converter in the temperature compensation module at room temperature. Ultrasonic velocity in the air was calculated at the current temperature. LED module will display the ranging distance results. The main program module can realize the initialization and control each program module and the hardware. The software block diagram of the system was showed as Fig. 4. 

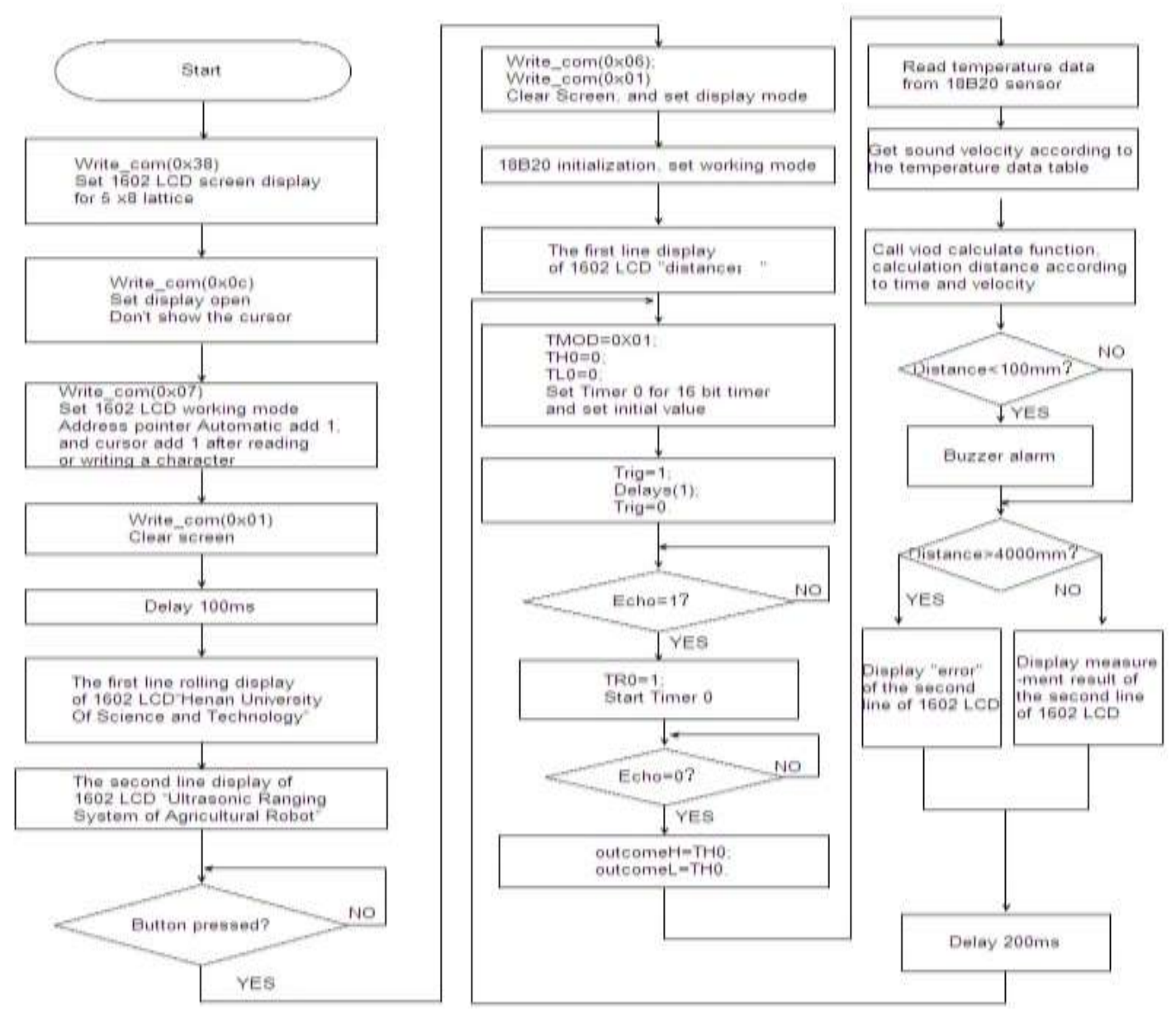

Fig. 4 The software block diagram of the system

\section{CONCLUSION}

The software system of ultrasonic ranging system in agricultural robot was designed. Ultrasonic ranging system software module structure mainly included main program module, the ultrasonic transmitting module, the interrupt service module of the comparator, the compensation module at room temperature and the display module. In the course of experiment, the software of ultrasonic ranging system with the hardware is simple, easy installation, and stably, and the ranging accuracy is higher, the scope of work completely meet the design requirements.

\section{ACKNOWLEDGEMENTS}

This work was supported by Scientific Research Foundation for Ph. Doctor of Henan University of Science and Technology.

\section{REFERENCES}

[1] Zhang Fu, Fu Sanling, Liu Yong, Xue Kunpeng and Zhang Yakun. The control system design of the wheel-legged robot. IOSR Journal of Engineering (IOSRJEN), 2(9), 2012,: 15-19.

[2] Zhang Fu and Fu Sanling. Hardware design of ultrasonic ranging system in agricultural robot. Advanced Materials Research, 2012, 430-432: 1167-1170

[3] Gao Yun. The Design of Ultrasonic Ranging System in Agriculture Robot. Journal of Agricultural Mechanization Research, (4), 2009: 76-79.

[4] Jinglei Tang, Xu Jing, Dongjian He and Feng David. Visual navigation control for agricultural robot using serial BP neural network. Transactions of the Chinese Society of Agricultural Engineering. 27(2), 2011: 194-198.

[5] Akihisa Ohya, Takayuki Ohno and Shin'ichi Yuta. Obstacle detectability of ultrasonic ranging system and sonar map understanding. Robotics and Autonomous Systems, 18(1-2),1996: 251-257.

[6] Ke-Nung Huang and Yu-Pei Huang. Multiple-frequency ultrasonic distance measurement using direct digital frequency synthesizers. Sensors and Actuators A: Physical, 149(1), 2009: 42-50.

[7] Wang Hongyun, Yao Zhimin, Wang Zhulin and Shi Lianyan. Design of Ultrasound Distance Measurement System. Instrumentation Technology, (11), 2010: 47-49. 